\section{Genetics and psychiatry ${ }^{\dagger}$}

\author{
MICHAEL J. OWEN and PETER McGUfFIN
}

Lionel Penrose, a psychiatrist who established his scientific reputation as a geneticist, once remarked that the two disciplines "make strange bedfellows" (Penrose, 1971). Over a quarter of a century later some readers of this journal would perhaps still agree. However, interest in this seemingly eccentric coupling has been greatly stimulated by the advent of molecular genetics, and this has led to both exaggerated optimism and unwarranted pessimism. Some see imminent advances in genetics as leading rapidly to new and more effective treatments for mental illness, whereas others see the prospect of discrimination, an assault on a cherished belief in free-will and perhaps a return to eugenics. In fact, both kinds of extreme view arise from misunderstandings of the potential power of genetics in psychiatry. As Rutter \& Plomin (1997) observe, a realistic appreciation of the likely. impact of genetic research on psychiatry can be achieved only once a number of these serious misconceptions have been put aside. Genetics has the potential to change and perhaps even transform psychiatry (Farmer \& Owen, 1996), but any transformation is likely to take time, and the challenge comes in understanding not simply genetic mechanisms, but also appreciating how they coact and interact with the environment.

\section{COMMON MISUNDERSTANDING}

Recently, the sterility of a nature-nurture debate has become obvious in the light of quantitative genetic studies using family, twin and adoption methods, and a consensus has begun to emerge that both genes and the environment are important in many mental illnesses and dimensions of normal behaviour. Quantitative geneticists have found it useful to describe the role of genes in terms of

tSee pp. 209-219, this issue. the proportion of variation in the population that is accounted for by genetic factors; they call this heritability. Unfortunately, as Rutter \& Plomin (1997) point out, the meaning of the term is often misunderstood. It is not a fixed property of a trait but is a feature of the population being studied. Therefore, it has no direct meaning at an individual level and, furthermore, its value may differ in different populations. For example, in a society where everybody smoked heavily, individual variation in the risk of getting lung cancer would be determined to a large extent by genetic factors, and the heritability would therefore be higher than in a society, like our own, in which there is more variability in smoking behaviour. However, in spite of the high heritability, the best way of reducing the incidence of lung cancer would be to modify the environment by persuading people to give up smoking. Another example of this phenomenon that is of relevance to psychiatry is phenylketonuria, a recessive disorder occurring in those with mutations in both copies of the phenylalanine hydroxylase gene. Given that phenylalanine is ubiquitous in our normal diet, this disorder has a heritability of $100 \%$. However, again it can be prevented by environmental manipulation: by removing phenylalanine from the diet. So if a disease has a high heritability, this means that genetic differences are important in influencing individual differences in disease susceptibility in the population. It does not on its own tell us whether modifications of the environment, either at a population or individual level, will be effective. Also, as Rutter \& Plomin (1997) remind us, it does not tell us the extent to which differences between populations are due to genetic or environmental differences.

Phenylketonuria is a comparatively rare condition caused by a defect in a single gene. However, it is clear from genetic research that the inheritance of common psychiatric disorders, like that of other common diseases, is complex and probably reflects the action of several or even many genes together with environmental factors. In other words, mental illness is not 'caused' or 'determined' by a person's genetic makeup and it is likely that each individual gene that is involved will play only a relatively small part in conferring susceptibility or predisposition. The failure to appreciate this is another major misconception about psychiatric genetics. It means that there is little prospect of geneticists identifying 'the gene for' schizophrenia, bipolar disorder, anxiety aggression, etc. Moreover, the alleles of genes conferring susceptibility will in some cases be quite common in the population and in some circumstances perhaps confer benefits rather than predispose to illness. This genetic complexity means that it is unlikely that the identification of the genes predisposing to mental illness will justify eugenic programmes or widespread termination of pregnancies. Moreover, genetic screening of the general population is unlikely to be particularly useful and the predictive value of detecting common susceptibility alleles is likely to be low.

\section{ADVANCES IN QUANTITATIVE GENETICS}

Having considered some of the things that genetics does not tell us, the reader might ask whether genetic research has told us anything useful about mental illness or behavioural characteristics. In fact, as Rutter \& Plomin (1997) show, quantitative genetics has made significant advances. For example, important genetic contributions to severe mental disorders such as schizophrenia, manic depression, recurrent depression and autism have been revealed (McGuffin $\boldsymbol{e t}$ $a l, 1994)$. Genes also seem to play a part in the milder 'neurotic' conditions, such as mild depression and anxiety and childhood disorders such as hyperactivity and specific reading disabilities. Substantial genetic influences have also been shown for dimensions of personality such as extraversion and neuroticism. Cognitive ability is another behavioural dimension showing a sizeable genetic influence (Plomin et al, 1994).

It is not often appreciated that this genetic research has another important implication: it provides some of the best evidence that the environment is important too, since genetic factors alone do not account for all of the observable variation in common behavioural traits and disorders.

There are still a number of areas in which more basic research using the methods of 
quantitative genetics is needed to delineate the role of genes. These include developmental and behavioural disorders of childhood, alcoholism, drug dependence and mild learning disability.

Advances are also being made in understanding how genes combine with specific environmental factors such as stressful lifeevents and social deprivation. Indeed, one of the most important contributions of quantitative genetics has been the demonstration that nature and nurture are not nearly so separate as was once assumed. Of direct relevance to psychiatry is the finding that the tendency of individuals with depression to report stressful life events is to some extent influenced by familial (McGuffin et al, 1988) and perhaps genetic factors (Kendler et al, 1993; Plomin et al, 1994; Thapar \& McGuffin, 1996). This may be so because those who are prone to depression are more likely to perceive events as threatening, or because they tend to expose themselves more to adversity. For example, an individual who (partly because of his genetic make-up) is impulsive, might make unsuitable choices of job or partner. Similarly those who are more 'sensation-seeking' (a moderately heritable trait) may be more prone to accidents. Such gene-environment correlations can also occur when genes conferring susceptibility to mental illness are passed on to a child, but also create via the parent apparently adverse environments because of such factors as impaired parenting and family discord.

\section{FINDING GENES}

In the past decade the most important development in psychiatric and behavioural genetics has been the widespread application of the techniques of molecular genetics to try to identify the genes involved. As we have seen, the inheritance of psychiatric disorders and behavioural variation is complex, reflecting multiple genes and environmental factors, and this has complicated attempts to locate and identify the relevant genes. However,

MICHAEL J. OWEN, FRCPsych, Divisions of Psychological Medicine and Medical Genetics; PETER McGUFFIN. FRCPsych, Division of Psychological Medicine, University of Wales College of Medicine, Cardiff

Correspondence: Professor Michael Owen, Neuropsychiatric Genetics Unit, Tenovus Building. University of Wales College of Medicine, Heath Park. Cardiff CF4 4XN. Tel: 01222743058 ; Fax: 01222 746554; e-mail: OwenMJ@Cardiff.ac.uk

(First received 14 May 1997, accepted 16 May 1997)

recent methodological developments together with the knowledge and technology provided by the Human Genome Project mean that we can now be reasonably confident that at least some of the genes contributing to common psychiatric disorders will be identified by the end of the first decade of the 21st century (Lander, 1996). It also seems likely that recently commenced work on the molecular genetics of behavioural traits and dimensions will begin to identify some of the genes involved in areas such as personality and cognitive abilities.

We agree with Rutter \& Plomin (1997) that the identification of susceptibility genes will ultimately be of great benefit to psychiatry, by leading to increased understanding of the disturbances of brain function that underlie mental disorders and the ways in which environmental factors interact with genetic vulnerability. This should pave the way for the development of new therapies which are better targeted at disease processes. Greater understanding of causation and mechanism should also aid diagnosis and open the door to targeted intervention strategies aimed at preventing the development or progression of disease in susceptible individuals and allowing specific treatments, whether pharmacological or psychological, to be better matched to specific diagnostic groups.

The journey from genes to therapies will involve many steps and will depend upon the ability of geneticists, neurobiologists, pharmacologists, psychologists and social scientists to work together. Fortunately steps are already being taken towards such an inte- grative approach (Brown, 1996; Mann \& Owen, 1996; Rutter \& Plomin, 1997) and we feel increasingly optimistic that the two sides of the old nature-nurture divide are beginning to seek common ground. It may not be possible to predict with certainty the extent and speed of advance, but we expect that no young psychiatrist completing his or her training now will find the next quarter century of their clinical practice uninfluenced by psychiatric genetics.

\section{REFERENCES}

Brown, G.W. (1996) Genetics of depression: a social science perspective. Internotional Review of Psychiatry. 8. 387-402.

Farmer, A. \& Owen, M. J. (1996) Genomics: the next psychiatric revolution? British fournal of Psychiatry, 169, 135-138.

Kendier, K. S., Neale, M., Kescler, R., ex ol (1993) A twin study of recent life events and difficulties. Archives of Generol Psychiatry, 50, 789-796.

Lander, E. S. (1996) The new genomics: global views of biology. Science. 274. 536-539.

McGuffin, P., Owen, M. J., O'Donovan, M. C., et al (eds) (1994) Seminars in Psychiatric Genetics. London: Gaskell.

_, Katz, R. \& Bebbington, P. (1988) The Camberwell Collaborative Depression: Study III. Depression and adversity in the relatives of depressed probands. British journal of Psychiotry. 152. 775-782.

Mann, A. H. Owen, M. J. (1996) The nature and nurture of depression: towards a new synthesis. International Review of Psychiotry, 8. 285-288.

Penrose, L. S. (1971) Psychiatric genetics. Psychological Medicine, I. 265-266.

Plomin, R., Owen, M. J. \& McGuffin, P. (1994) The genetic basis of complex human behaviours. Science, 264, 1733-1739.

Rutter, M. A Plomin, R. (1997) Opportunities for psychiatry from genetic findings. British journal of Psychiatry, 17. 209-219.

Thapar, A. McGufiln, P. (1996) Genetic influences on life events in childhood. Psychological Medicine, 26. 813-820. 